\title{
Editorial
}

\section{Enabling Technologies for Fifth-Generation Mobile Communications}

\author{
Jianhua Zhang, ${ }^{1}$ Chih-Lin I, ${ }^{2}$ Juho Lee, ${ }^{3}$ Jungnickel Volker, ${ }^{4}$ Jilei Hou, ${ }^{5}$ and Sujit Dey ${ }^{6}$ \\ ${ }^{1}$ Beijing University of Posts and Telecommunications, Beijing 100876, China \\ ${ }^{2}$ China Mobile Research Institute, Beijing 100053, China \\ ${ }^{3}$ Samsung Electronics Co., Ltd., Suwon-si, Gyeonggi-do 443-742, Republic of Korea \\ ${ }^{4}$ Fraunhofer Heinrich Hertz Institute, 10587 Berlin, Germany \\ ${ }^{5}$ Qualcomm Inc., 5775 Morehouse Drive, San Diego, CA 92121, USA \\ ${ }^{6}$ Department of Electrical and Computer Engineering, University of California at San Diego, \\ San Diego, CA 92093, USA \\ Correspondence should be addressed to Jianhua Zhang; jhzhang@bupt.edu.cn
}

Received 30 March 2016; Accepted 30 March 2016

Copyright (C) 2016 Jianhua Zhang et al. This is an open access article distributed under the Creative Commons Attribution License, which permits unrestricted use, distribution, and reproduction in any medium, provided the original work is properly cited.

ITU-R has named the fifth-generation (5G) mobile communications as IMT 2020 and described its vision from eight aspects, including peak data rate, mobility, connection density, and delay. To meet the continuously increasing high data rates demand and new applications scenarios, some emerging technologies such as massive MIMO, 3dimensional (3D) MIMO, millimeter wave (mmWave), and new network architecture are being investigated and studied. It is foreseeable that these efforts from both academia and industry will make 5G realize the vision: "Information a finger away, everything in touch."

This special issue contains several papers selected from submissions. These papers highlight some of the current research interests and achievements in the area of enabling technologies for fifth-generation mobile communications. The paper " $5 \mathrm{G}$ : Vision and Requirements for Mobile Communication System towards Year 2020,” by G. Liu and D. Jiang, described the vision of $5 \mathrm{G}$ and summarized some candidate technologies and spectrum solutions.

The channel state information acquisition at the transmitter is a great challenge in frequency-division duplex (FDD) massive MIMO systems. The paper "Multiuser Beamforming with Limited Feedback for FDD Massive MIMO Systems," by S. Zheng et al., discussed the multiuser beamforming in FDD massive MIMO systems and proposed a novel multiuser beamforming scheme.

By taking the elevation domain into consideration, the 3D channel model gives a better understanding of practical channel statistical characteristics. The paper "Statistical Characteristics of Measured 3-Dimensional MIMO Channel for Outdoor-to-Indoor Scenario in China and New Zealand," by Y. Yu et al., presented a comparative result based on 3D MIMO channel measurements performed in both China and New Zealand.

For the study of propagation channel characteristics at mmWave frequency band, the paper "Propagation Channel Comparison between 23.5 and $45 \mathrm{GHz}$ in Conference Scenario," by J. Dou et al., proposed a hybrid channel modeling approach considering both deterministic simulation and measurement-based analysis and established the statistical channel model for both the large and small scale characteristics at 23.5 and $45 \mathrm{GHz}$.

In $5 \mathrm{G}$ mobile network, various network mechanisms coexist and complement each other to provide ubiquitous high speed wireless connectivity, resulting in a new bottleneck of universal access. The paper "Research on Joint Handoff Algorithm in Vehicles Networks," by Y. Bi et al., 
provided an advanced dynamic handoff algorithm in vehicles networks to solve the seamless handoff problem.

\title{
Acknowledgments
}

We would like to thank all authors for contributing papers to this special issue.

\author{
Jianhua Zhang \\ Chih-Lin I \\ Juho Lee \\ Jungnickel Volker \\ Jilei Hou \\ Sujit Dey
}




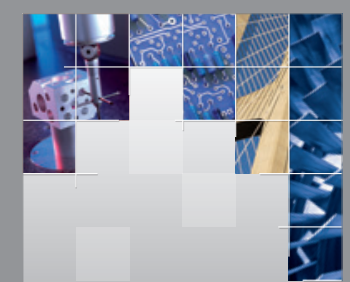

\section{Enfincering}
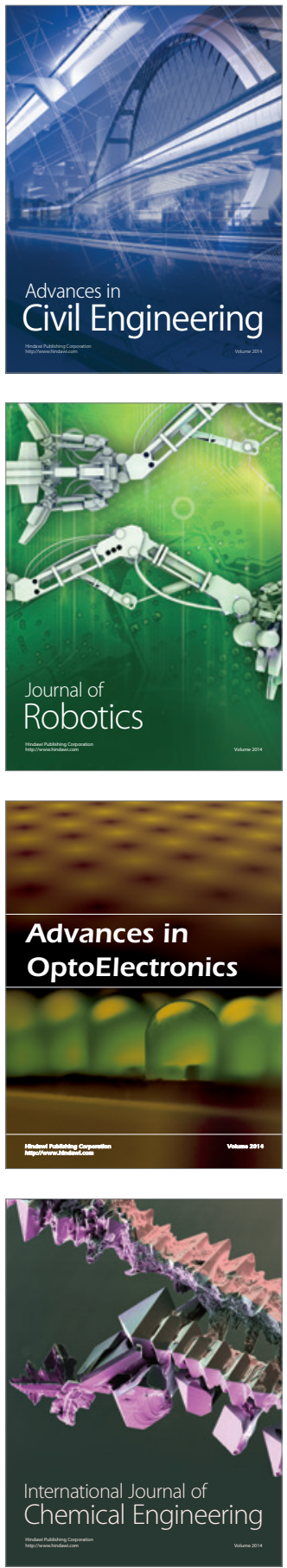

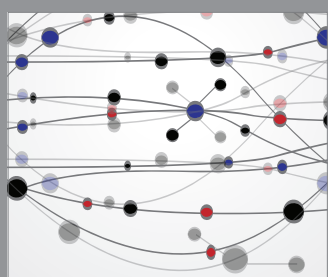

The Scientific World Journal

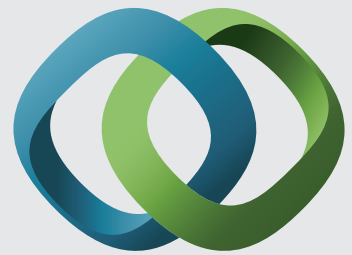

\section{Hindawi}

Submit your manuscripts at

http://www.hindawi.com
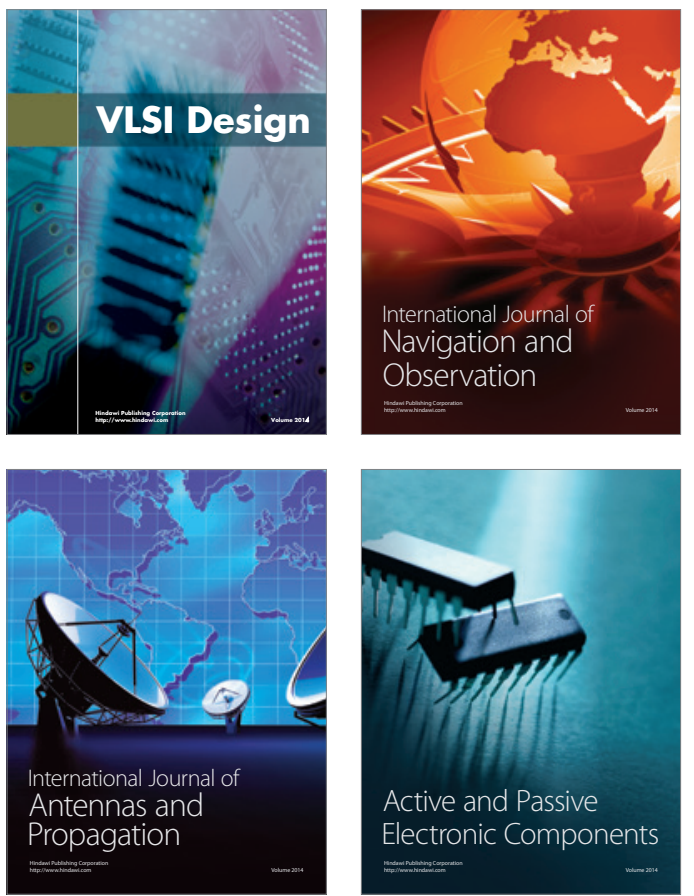
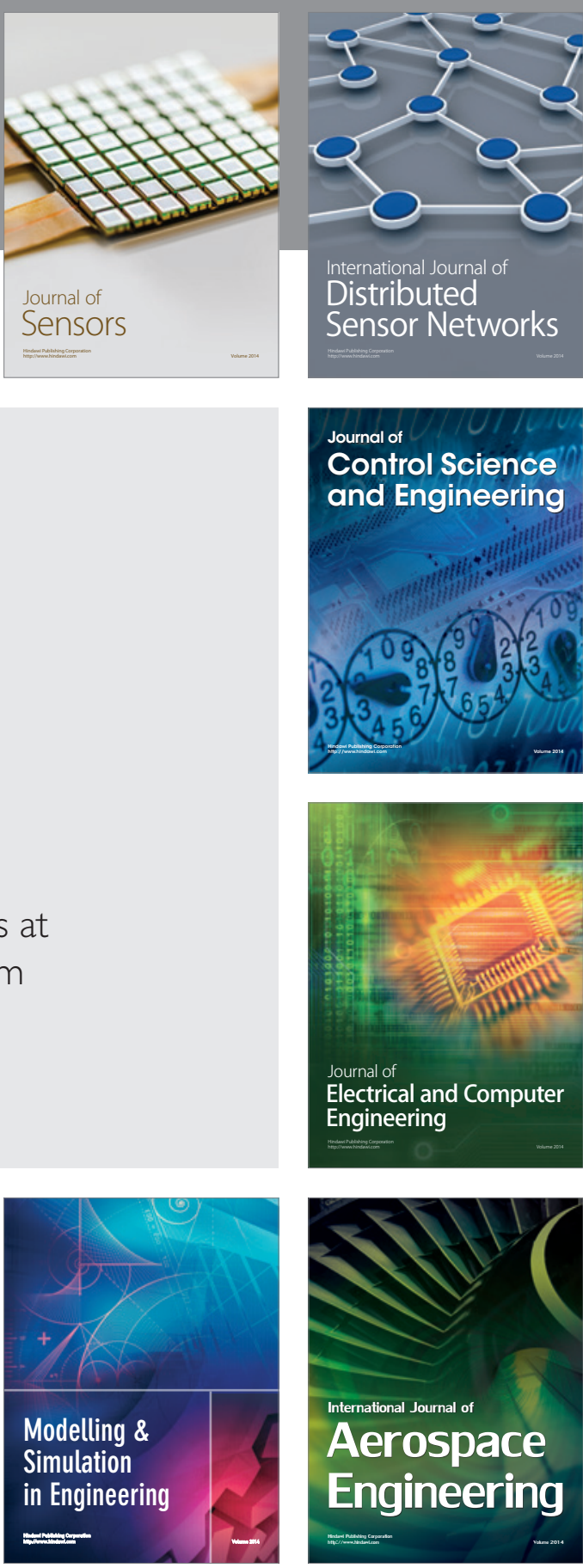

International Journal of

Distributed

Sensor Networks

Journal of

Control Science

and Engineering
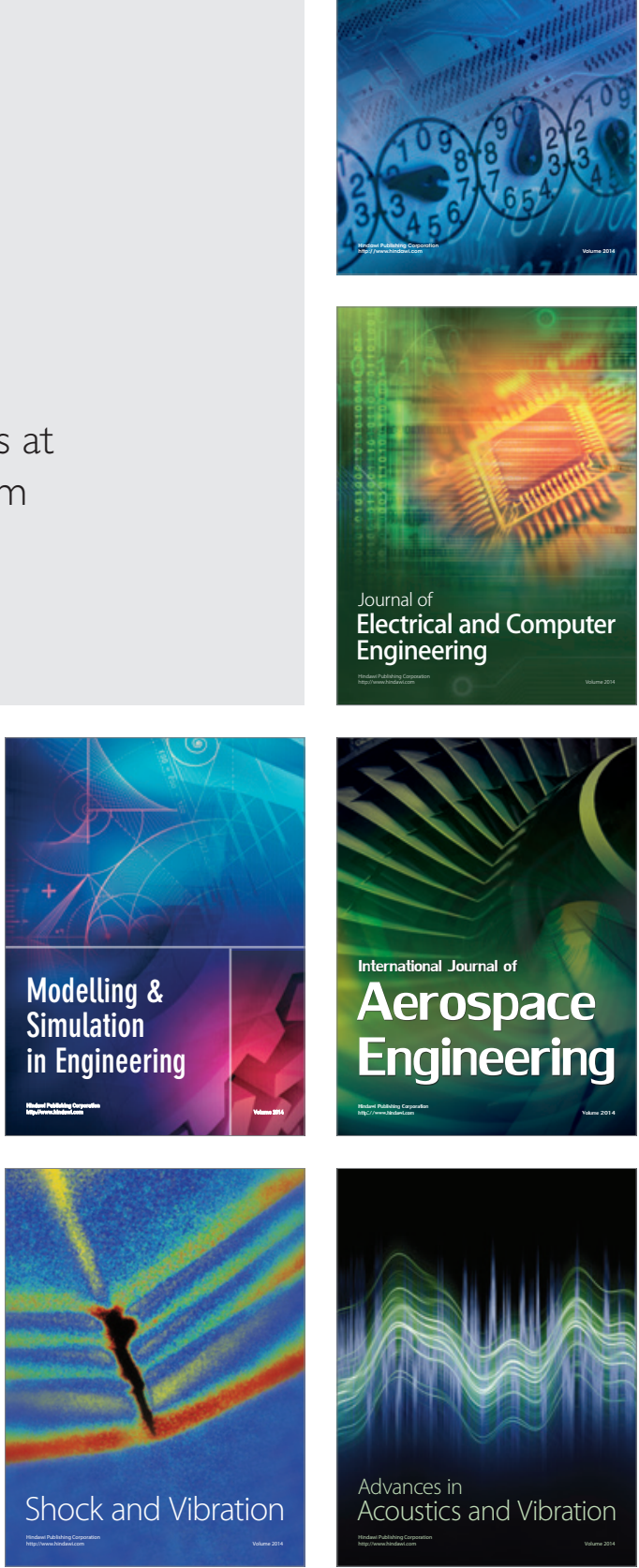